\title{
Le réabonnement à un système de paniers : \\ Le cas des produits biologiques solidaires
}

Pour citer cet article :

Jara M., Dufeu I., Le Grel L. (2020), Mesure des facteurs de réabonnement à un système de paniers: Le cas des produits biologiques solidaires. Décisions Marketing, oct-nov, 100. (HCERES B, FNEGE 3). DOI : 10.7193/DM.100.97.116

\section{Résumé :}

Les formules de paniers de produits agricoles locaux se développent ces dernières années en France. Le succès apparent des Paniers Bio Solidaires (PBS) masque un problème de réabonnement des adhérents. Cette recherche vise à explorer les profils des adhérents puis tester plus largement les facteurs pouvant favoriser le réabonnement à un système de paniers de fruits et légumes biologiques et locaux. Les résultats montrent que (1) les abonnés présentent des profils différenciés entre eux (2); l'engagement et la satisfaction représentent les facteurs déterminants de l'intention comportementale; (3) les changements d'habitudes alimentaires n'expliquent pas la prédisposition à s'engager durablement.

Mots clés : système de paniers, intention comportementale, engagement, consommation biologique, typologies.

\begin{abstract}
:
The basket purchases of organic and local agricultural products are developing in recent years in France. The success of one of them entitled "Paniers Bio Solidaires" (PBS) hides a problem of re-subscription of members. This research aims to explore and to test more broadly the factors leading to maintain the subscription to a basket system. The results show that (1) members have differentiated profiles; (2) commitment and satisfaction are the main determinants of behavioral intentions; (3) changes in eating habits do not build commitment.
\end{abstract}

Keywords: basket purchase, behavioral intentions, commitment, organic consumption, cluster analyses. 


\section{Introduction}

En 2018, trois quarts des Français consomment du bio au moins une fois par mois. Pour $16 \%$ des Français, c'est même un acte quotidien ${ }^{1}$; chaque année la proportion de nouveaux consommateurs augmente rendant ainsi ce marché très attractif pour les distributeurs. Aujourd'hui, deux options de distribution (non exclusives) s'offrent aux consommateurs : les circuits traditionnels tels que la grande distribution et la distribution spécialisée (Biocoop par exemple), les circuits alternatifs reposant sur un modèle social et solidaire tels que les Amap (Associations pour le maintien de l'agriculture paysanne).

À la suite du succès des Amap depuis le début des années 2000, système reposant sur un engagement réciproque fort entre agriculteurs locaux et consommateurs dans un cadre associatif, des formules dérivées et moins engageantes se sont multipliées. Ces systèmes, majoritairement issus de l'agriculture biologique, reposent sur des principes de fonctionnement variés (avec plus ou moins de services rendus au consommateur : livraison à domicile ou en point relais, contenu ajustable de façon hebdomadaire ou déterminé par la production, engagement sur plusieurs mois ou paiement par panier, etc.) Les Amap ont ceci de très particulier qu'elles reposent sur une implication forte des consommateurs (appelés mangeurs) allant jusqu'à la coordination, la communication et la distribution.

Les autres systèmes sont au contraire majoritairement organisés par les producteurs (les paniers bio, les drives fermiers...) ou par des organisations jouant le rôle d'intermédiaire organisateur (La Ruche qui dit oui, par exemple). Le «mangeur» y est moins actif et choisit simplement l'offre qui lui convient le mieux. C'est le cas du système étudié dans cette recherche, les Paniers Bio Solidaires (PBS). Cette offre s'inscrit dans le champ de l'économie sociale et solidaire mais place le « mangeur » dans un rôle de simple consommateur.

Les formules de paniers impliquent toutes une contrainte particulièrement forte pour les acteurs : le besoin de prévisibilité. L'organisation de ces filières suppose en effet une capacité d'anticipation importante. Les producteurs et les éventuels intermédiaires ou coordinateurs

1 https://www.lsa-conso.fr/qui-sont-les-consommateurs-bio,227835. 
ont besoin de s'inscrire dans un horizon temporel assez long, afin de dimensionner le système: planifier les mises en production (voire intégrer de nouvelles cultures afin de compléter les paniers) et prévoir l'approvisionnement, la logistique et la distribution qui sont propres à ces systèmes (surtout lorsque les paniers sont acheminés vers le consommateur ou vers des points relais).

Dans ce contexte, une incertitude sur les réabonnements à la période suivante est particulièrement dommageable, d'autant plus que le marché du bio en phase de croissance (+12\% par an environ) incite de plus en plus d'acteurs à proposer une offre compétitive et à détourner potentiellement les adhérents de paniers. La pression concurrentielle est donc forte et menace ce système commercial spécifique. Ce questionnement managérial est concrètement vécu par les Paniers Bio Solidaires (PBS). Le succès en effet apparent des PBS, attesté par sa forte croissance annuelle (avec désormais plus de 2000 paniers livrés chaque semaine) et par des niveaux de satisfaction déclarée élevés (moyenne $=4,24 / 5$ ), masque un problème récurrent depuis le début : un taux de départ élevé. En effet, chaque année 25 à $30 \%$ des adhérents ne renouvellent pas leur abonnement ${ }^{2}$. Autrement dit, la satisfaction comme les perceptions positives ne suffisent pas à retenir les abonnés.

Certains chercheurs se sont particulièrement emparés de la question des systèmes de paniers pour les étudier sous l'angle du marketing relationnel. Ils ont ainsi travaillé sur les comportements et les représentations des consommateurs (Curtis, Allen et Ward, 2015 ; Robert-Demontrond, Beaudouin et Dabadie, 2017) et mis en évidence le rôle joué par la confiance (Aissaoui, Bueno Merino, et Grandval, 2017) et l'engagement (Dufeu et Ferrandi, 2013) dans le cas particulier des Amap. Mais cette littérature ne s'est pas à notre connaissance intéressée à la question des déterminants de la fidélisation des adhérents des systèmes de paniers.

\footnotetext{
${ }^{2}$ Données internes de l'association.
} 


\section{Encadré 1 : Les Paniers Bio Solidaires (PBS)}

L'association Paniers Bio Solidaires (PBS) est née en 2010 de l'alliance de producteurs bio engagés dans une démarche de commerce équitable local et de structures d'insertion de personnes en difficultés sociales. Les PBS livrent en points relais (divers types de points de vente dans des villes des Pays-de-la-Loire) de façon hebdomadaire des paniers de fruits et légumes bio et locaux produits par les adhérents de Bio Loire Océan (BLO - une association de producteurs de fruits et légumes des Pays-de-la-Loire). Le prix de chaque panier est de 13,90 euros (avec 5 à 7 variétés pour 3,5 kg environ). Les adhérents des PBS s'abonnent pour une saison et, contrairement aux Amapiens, ne participent pas à la distribution. Le site internet est le principal médium, fortement utilisé tant par l'association que par les adhérents qui consultent la composition du panier à venir, les idées de recettes et les informations sur les producteurs et sur les produits ${ }^{3}$.

La présente recherche, en s'inscrivant dans le champ théorique de la fidélité en marketing, répondra plus précisément aux questions suivantes :

1) Existe-t-il des profils spécifiques de consommateurs de paniers bio locaux ? Autrement dit, les abonnés des paniers sont-ils homogènes dans leurs habitudes alimentaires, pratiques, perceptions de l'offre et intentions comportementales (de réabonnement) ? Du point de vue empirique, quelles méthodes de recherche mobiliser pour révéler ces profils différenciés ?

2) Les déterminants majeurs des intentions comportementales envers le système de paniers sont-ils différents de ceux qui ont été mis à jour pour les circuits de distribution plus conventionnels?

3) Les comportements alimentaires, identifiés comme critères de segmentation, peuvent-ils jouer sur le niveau d'engagement envers un système de paniers?

Les PBS, en s'appuyant sur une logique de développement durable et de commerce équitable local et de promotion de méthodes d'agriculture biologiques très exigeantes, constituent un cadre convenable pour nous permettre d'explorer et de proposer des éléments de réponse à ces

3 https://www.lespaniersbiosolidaires.fr/concept-paniers-bio 
différentes questions. La base de données constituée de consommateurs réels puisqu'il s'agit des abonnés des PBS (800 répondants à un questionnaire reprenant la plupart des variables du marketing relationnel et leurs échelles de mesure) assurera la robustesse des résultats empiriques.

Après avoir présenté le cadre théorique de cette recherche, qui renvoie au concept de fidélité en marketing, cet article expose dans une seconde partie la conduite d'analyses typologiques étudiant de manière affinée les profils d'abonnés du portefeuille. Ces analyses descriptives révéleront une hétérogénéité du portefeuille d'abonnés selon leurs habitudes alimentaires, leurs perceptions de l'offre et leur niveau d'intention comportementale (calculé par un score d'intention de réabonnement). Dans une troisième partie des analyses confirmatoires seront à même de tester le pouvoir explicatif des changements (ou pas) des habitudes alimentaires sur la prédisposition à s'engager durablement avec le système PBS. Les résultats de cette recherche permettront une prise de recul sur les spécificités de profils des abonnés à un système de paniers (différences entre eux ; différences et points communs avec les clients des circuits de distribution alimentaires traditionnels), d'apprécier plus finement les déterminants des intentions comportementales positives à un système de paniers, de questionner la notion d'engagement durable et de faire émerger finalement des propositions commerciales adaptées à chaque profil d'abonnés.

\section{La fidélité des consommateurs de paniers bio locaux}

La littérature scientifique sur les circuits courts alimentaires s'est intéressée depuis une dizaine d'années aux perceptions/représentations mentales et comportements des consommateurs (voir par exemple Merle et Piotrowski, 2012 ; Curtis, Allen et Ward, 2015 ; Robert-Demontrond, Beaudouin et Dabadie, 2017) mais sans aborder spécifiquement le concept de fidélité. 


\section{Fidélité attitudinale $v s$ comportementale}

Les travaux en marketing ont montré que le concept de fidélité des individus (loyalty en anglais) pouvait être abordé sur le plan comportemental - «le comportement est l'expression même de la fidélité » (Tucker, 1964), mesuré à travers les achats répétés/réachats (Frisou, 2004) ou l'intention de ré-achat (Chaudhuri et Holbrook, 2001) ; ou sur le plan attitudinal - il s'agit d'une prédisposition du consommateur. La fidélité est présente chez le client avant même de passer à l'acte (Jacoby et Kyner, 1973 ; Lichtlé et Plichon, 2008 ; Raïes et GavardPerret, 2011).

La fidélité attitudinale est le plus souvent considérée comme un antécédent significatif de la fidélité comportementale (ibid ; Lichtlé et Plichon, 2008). La relation entre les deux concepts est cependant l'objet de controverses en marketing; la présente étude se centre sur l'attitude des répondants et sur ses déterminants, sans préjuger de leurs comportements réels dans le futur. La fidélité attitudinale représente le socle fondateur prédisposant le consommateur à construire et entretenir une relation durable ; c'est pourquoi, il nous semble à la fois pertinent et déterminant de débuter par son étude.

\section{Les déterminants de la fidélité attitudinale}

Une série de travaux relevant d'une perspective relationnelle montre que la dimension affective soutient la relation durable entre les parties (Lichtlé et Plichon, 2008 ; Rivière et Mencarelli, 2012). D’une manière générale, elle révèle que les intentions comportementales des consommateurs sont déterminées par deux facteurs majeurs :

1) la confiance (crédibilité, bienveillance et intégrité de la marque d'après Gurviez et Korchia, 2002, repris par Cissé-Depardon et N'Goala, 2009),

2) l'engagement (Morgan et Hunt, 1994 ; Lacœuilhe, 2000 ; Cissé-Depardon et N'Goala, 2009 ; Raïes et Gavard-Perret, 2011).

Les recherches récentes qui se sont penchées sur le marketing relationnel dans un contexte alimentaire, confirment l'importance de la confiance et de l'engagement et notamment dans le cas des systèmes alimentaires alternatifs (voir par exemple Hérault-Fournier, 
Merle et Prigent-Simonin, 2014 ou Dufeu et Ferrandi, 2013). Elles révèlent aussi l'importance d'une autre dimension, de nature à la fois affective et cognitive : la proximité ressentie (ibid ; Bergadaà et Del Bucchia, 2009 ; Labbé-Pinlon, Lombart et Louis, 2016 ; Merle, Hérault-Fournier et Werle, 2016). La proximité ressentie recouvre notamment les quatre dimensions suivantes ${ }^{4}$ que nous retenons dans cette recherche (et qui ont généré les items du questionnaire) :

- la proximité axiologique (ou identitaire chez Bergadaà et Del Bucchia, 2009) se construit sur les valeurs que représente le producteur ou le distributeur aux yeux de l'acheteur ;

- la proximité relationnelle se noue autour des relations directes et répétées qu'entretiennent des individus, ici l'acheteur avec les producteurs et distributeurs ;

- la proximité de processus renvoie au niveau de connaissance qu'a l'acheteur des processus mis en œuvre par le distributeur ou le producteur.

- la proximité d'accès (proche de l'idée de proximité géographique mais avec l'idée de perception d'une plus ou moins grande facilité d'accès).

De manière complémentaire, la satisfaction est souvent citée comme l'antécédent majeur de la fidélité. Depuis les années 2000, plusieurs articles ont montré que la satisfaction n'explique pas bien la fidélité attitudinale (Bennett et Rundle-Thiele 2004). Oliver (1999) défendait en effet que la satisfaction participe à la création d'un lien durable - la fidélité - mais n'est pas une condition suffisante à elle seule. Un consommateur satisfait est fidélisable - c'est un prérequis de fidélité attitudinale. Néanmoins, il arrive que le client ne revienne pas systématiquement ou ne construise pas de manière durable un lien avec l'organisation (Michael et al., 2008). Autrement dit, même des consommateurs satisfaits peuvent quitter une organisation (Jones et Sasser, 1995). En conclusion, la satisfaction est un bon indicateur mais est insuffisant pour construire une relation durable avec le client. D'autres variables doivent

\footnotetext{
${ }^{4}$ Qui reprennent l'essentiel des dimensions considérées dans les études récentes en marketing, à l'exception de la proximité fonctionnelle (Bergadaà et Del Bucchia, 2009); nous mesurons cependant bien dans cette étude l'évaluation par les adhérents des caractéristiques matérielles de l'offre.
} 
s'ajouter pour construire cette relation à long terme. L'encadré 2 présente l'ensemble des variables et items de mesure de cette recherche.

\section{Encadré 2 : Design de la recherche}

Le questionnaire a été construit en collaboration entre les auteurs de cet article et les gestionnaires des PBS. Ces derniers avaient déjà conduit plusieurs enquêtes de satisfaction (une par an depuis 2011) et échangé (par téléphone souvent) avec une grande partie des premiers abonnés. Ils connaissaient donc bien les dimensions de la valeur perçue et les facteurs d'(in)satisfaction, ainsi que le profil de leurs abonnés. Les chercheurs ont pu analyser les résultats de ces enquêtes avant de proposer un enrichissement du questionnaire en intégrant les dimensions repérées dans la littérature exposée précédemment. Les échelles de mesure de cette recherche ont été validées statistiquement (l'annexe la expose les tests de fiabilité de ces mesures). Le questionnaire présente 31 questions (détaillées en annexe 1b) et est entièrement évalué en échelle de Likert (5 échelons allant de «pas du tout d'accord» à «tout à fait d'accord» avec la proposition citée). Les thèmes du questionnaire qui seront mobilisés pour cette étude sont essentiellement :

1) l'évaluation de la satisfaction cumulée des variables commerciales (produits, prix, point relais tiré de Cissé-Depardon et N'Goala, 2009);

2) la confiance (concept mesuré par Gurviez et Korchia, 2002 et repris par Cissé-Depardon et N'Goala, 2009 - «j'ai confiance en la qualité des PBS », « les PBS sont à mon écoute », « les PBS sont sans danger » correspondant à la dimension de crédibilité de Gurviez et Korchia, 2002);

3) l'engagement (« je parle des PBS autour de moi, je trouverais difficile de quitter les PBS », « je reviendrais vers les PBS si je devais cesser mon abandonnement quelque temps », « je suis attaché aux PBS - je suis très lié aux PBS »- tiré de Lacœuilhe, 2000 et Cissé-Depardon et N'Goala, 2009);

4) les proximités (axiologique, relationnelle et de processus), tirées de Hérault-Fournier et al., 2014 : «je connais les valeurs des PBS", "je partage plus de valeurs avec les PBS qu'avec 
d'autres magasins, mes valeurs sont les mêmes que celles des PBS »; « mieux connaître les producteurs », « rencontrer les producteurs »; «je sais d'où proviennent les fruits et légumes des PBS », «j'ai accès aux informations concernant la production, la préparation et la distribution des paniers », « je connais le mode de production des PBS », « les PBS pourraient me satisfaire davantage en me permettant d'avoir plus d'information sur les produits », «les PBS pourraient me satisfaire davantage en me permettant d'avoir plus d'informations sur les personnes en réinsertion") ;

5) l'intention de se réabonner («avez-vous l'intention de vous réabonner la saison prochaine ?»).

Sur une population mère de plus de 2000 adhérents tous sollicités par courriel, 858 ont répondu. Suite au retrait des questionnaires incomplets ou présentant des réponses aberrantes, 825 questionnaires ont été conservés. L'échantillon composé de 825 abonnés recouvre une population majoritairement féminine ( $80 \%$ ), jeune (moyenne autour de 35 ans), vivant en famille.

\section{Des intentions comportementales différentes selon le profil des abonnés}

Après avoir précisé le cadre théorique de cette recherche, il s'agit à présent de répondre aux questions suivantes : existe-t-il des profils spécifiques de consommateurs de paniers bio ? Autrement dit, les abonnés des PBS sont-ils homogènes dans leurs habitudes, pratiques et intentions comportementales (de réabonnement)?

Pour ce faire, la mobilisation d'analyses typologiques, comme méthodes de recherche, s'avère ici pertinente car elles permettent de révéler l'existence de différences entre les abonnés dans leurs intentions comportementales.

Auparavant les caractéristiques de l'échantillon seront exposées. 


\section{Caractéristiques de l'échantillon}

Les répondants de cette enquête sont abonnés depuis un an en moyenne (c'est également la médiane, un quart de l'échantillon étant abonné depuis plus de deux ans). Les principaux critères de choix des produits alimentaires sont la saisonnalité et l'origine géographique ; suivent, dans une moindre mesure, le caractère biologique, le prix, la fraicheur puis le goût. Ces critères sont très conformes aux autres études sur les consommateurs en circuits courts locaux en France (Merle et Piotrowski, 2012 ; Mundler, 2013 ; Hérault-Fournier, Merle et Prigent-Simonin, 2014).

La moitié des répondants consommait de manière significative des fruits et légumes bio avant d'adhérer mais ils étaient très rarement des adeptes des circuits alternatifs et très rarement de systèmes de paniers. Plus de la moitié d'entre eux achetaient leurs fruits et légumes majoritairement en supermarché et un tiers environ sur des marchés de plein vent. Seuls $5 \%$ fréquentaient des Amap ou autres systèmes de paniers. Ils n'ont donc pas un profil militant à l'origine. D'ailleurs, les consommateurs ont majoritairement connu le système par des prospectus distribués dans leur quartier.

Il apparaît de plus que la valorisation de l'offre des PBS est forte : plus de $90 \%$ se disent satisfaits du goût, de la fraicheur et de la tenue des produits, de la présentation du panier, de la praticité du point relais, de la gestion des abonnements, du site internet et de la fréquence de distribution. Le prix est enfin jugé raisonnable pour $60 \%$ des adhérents, «plutôt cher » pour $25 \%$ et «plutôt pas cher» pour $12 \%$. Il parait donc risqué d'augmenter le prix des paniers. La satisfaction cumulée (sur l'ensemble des variables commerciales) est quant à elle évaluée à $4 / 5$ par $40 \%$ des répondants et $5 / 5$ par $55 \%$ des répondants. Le niveau global s'élève à $4,24 / 5$.

Les questions mesurant la proximité ressentie (Bergadaà et Del Bucchia, 2009 ; HéraultFournier, Merle et Prigent-Simonin, 2014) avec les PBS ont révélé une appréciation très positive pour la proximité axiologique (80\% environ selon les variables manifestes). La proximité de processus affiche de moins bons résultats : une proportion non négligeable (39\%) dit vouloir plus d'informations sur les produits et les personnes qui produisent et font vivre le système. La confiance dans la qualité des produits et la sécurité des aliments est quasi 
unanime ; l'intégrité, la bienveillance et l'honnêteté des PBS à leur égard (Cissé-Depardon et N'Goala, 2009) sont très élevées (personne ou presque n'exprime de défiance). Dès lors, il apparaît logique que l'engagement, qui traduit le désir de l'individu de poursuivre et de valoriser la relation sur le long terme soit fort en moyenne. C'est vrai pour la dimension affective de l'engagement (je suis attaché aux PBS) : $60 \%$ environ se sentent «plutôt » ou «tout à fait engagés » affectivement (selon les variables manifestes de l'échelle de mesure adaptée de Cissé-Depardon et N'Goala, 2009 et Raïes et Gavard-Perret, 2011). C'est aussi vrai pour l'intention de réabonnement positive à $83 \%$ et le bouche à oreille à $90 \%$, (ibid).

\section{Des profils différenciés selon leurs habitudes alimentaires, leurs perceptions de l'offre et leur intention comportementale}

L'analyse typologique implique la conduite au préalable d'analyses en composantes principales (ACP). Ces analyses exploratoires ont pour objectif, d'une part, d'épurer définitivement l'ensemble des items de mesure (questions) problématiques (car non reliés statistiquement au phénomène) et d'autre part, de structurer les restants, en composantes. C'est ainsi que les 31 questions (après épuration) se structurent en six composantes ${ }^{5}$ (restituant 52,65\% de l'inertie totale du jeu de données, tableau 1). La première composante en restitue $14,63 \%$; cette composante principale est fortement corrélée aux variables révélant une dimension relationnelle (confiance, engagement et proximité axiologique). La seconde $(10,34 \%$ de l'inertie) est essentiellement corrélée aux changements des habitudes alimentaires grâce aux PBS. La troisième composante $(8,26 \%)$ est corrélée à des aspects fonctionnels liés à la distribution (point relais), la quatrième aux proximités relationnelle et de processus $(7,17 \%)$ et la cinquième à la perception du prix $(6,47 \%)$. Une sixième composante enfin est portée par la composition familiale $(5,78 \%)$.

5 Une rotation Varimax est lancée en vue d'augmenter la lisibilité des composantes. 
dma190087 R3

Tableau 1 : Les profils d'adhérents selon les six composantes de l'ACP

\begin{tabular}{lcccccc}
\hline Classe & $\begin{array}{c}\text { Confiance, proximité } \\
\text { axiologique, } \\
\text { engagement et ravis }\end{array}$ & $\begin{array}{c}\text { Changement } \\
\text { d'habitudes grâce au } \\
\text { bio inconnu avant }\end{array}$ & $\begin{array}{c}\text { Point } \\
\text { relais }\end{array}$ & $\begin{array}{c}\text { Proximités } \\
\text { relationnelle et de } \\
\text { processus }\end{array}$ & $\begin{array}{c}\text { Prix } \\
\text { perçu } \\
\text { élevé }\end{array}$ & $\begin{array}{c}\text { Composition } \\
\text { familiale }\end{array}$ \\
\hline 1 Les conventionnels (347 ind.) & $-0,141$ & 0,001 & $-0,778$ & 0,086 & $-0,222$ & 0,074 \\
2 Les expérimentés (213 ind.) & 0,439 & $-0,206$ & 0,430 & $-0,671$ & $-0,354$ & 0,231 \\
3 Les expérimentateurs (265 ind.) & $-0,042$ & 0,520 & 0,346 & 0,146 & 0,338 & $-0,996$ \\
\hline
\end{tabular}


1 Dans un second temps, des analyses typologiques (encadré 3) sont lancées à partir des six 2 composantes de l'ACP. La classification typologique a posteriori (classification ascendante 3 hiérarchique, encadré 2) révèle trois groupes différents d'abonnés. L'étude des objets centraux 4 permet d'obtenir une lecture précise du profil de chaque groupe et ainsi de les nommer (groupe 1 - «les expérimentés »; groupe 2 - «les expérimentateurs »; groupe 3 - «les conventionnels »).

Encadré 3 : point méthodologique sur les typologies

8 Cette technique permet de discriminer l'échantillon sur la base d'axes générés préalablement 9 par une analyse en composantes principales exploratoire. La typologie a pour objectif de réduire la dispersion des individus de l'étude en les regroupant dans des classes (groupes ou 11 types) homogènes et différenciées.

La typologie ascendante hiérarchique $(\mathrm{CAH})$ vise en particulier à regrouper, a posteriori, les individus en groupes à la fois hétérogènes entre eux (du point de vue des perceptions et des motivations) et homogènes à l'intérieur de chacun. L'arbre de classification (ou dendrogramme - annexe 2) permet de visualiser la succession de regroupements entre les marques. Lorsque la perpendiculaire obtenue coupe les « branches » de l'arbre, le nombre de groupes à retenir apparaît. Ici, une typologie en trois groupes semble convenir.

À l'issue de l'analyse, pour chacun des trois groupes formés, un modèle causal est testé en vue de mesurer l'intention de se réabonner à travers la variable manifeste « lors de la prochaine saison, avez-vous l'intention de vous réabonner» (réponse sur une échelle de Likert de 1 à 5). De là, un score d'intention de réabonnement est calculé pour chaque groupe.

22 Groupe 1 - «les conventionnels » (347 individus) : un premier groupe se dégage, constitué de 347 individus marqués par des spécificités sur le plan des attentes fonctionnelles. Au regard des objets centraux présentés dans le tableau 1, ces individus sont fortement et négativement représentés sur l'axe 3. Autrement dit, ils perçoivent fortement et négativement le système de distribution proposé. Nous les nommons «les conventionnels» illustrant clairement des abonnés à la recherche d'un bénéfice fonctionnel (préférant donc un système de distribution rapide et pratique d'accès). 
1 Après avoir validé la fiabilité de chaque variable du modèle, l'intention de se réabonner pour

2 les «conventionnels" s'écrit: Intention de se réabonner $=0,412$ engagement $+0,370$

3 satisfaction $+0,062$ confiance $-0,040$ proximité de processus.

$4 \quad$ La figure 1 illustre ce modèle causal.

5 Figure 1 - Modèle causal des intentions de se réabonner des «conventionnels »

6

8 L'engagement et la satisfaction cumulée (sur les variables commerciales) restent

9 prioritairement les facteurs construisant cette intention. La dimension relationnelle (à travers 10 la confiance) joue un rôle beaucoup plus faible mais significatif dans l'intention de se 11 réabonner. Les dimensions fonctionnelles et relationnelles de la consommation caractérisent 12 donc ce groupe.

13 En calculant la moyenne de chaque variable manifeste composant le modèle causal des 14 « conventionnels », nous obtenons le score d'intention de réabonnement suivant : 2,65/4,02 15 soit $66 \%{ }^{6}$ (écart de 1,37 - soit $34 \%$ ).

6 4,02 correspond au score maximal du modèle c'est-à-dire lorsque toutes les variables obtiennent la note de 5/5 sur l'échelle de Likert. 
1 Groupe 2 - «les expérimentés » (213 individus). Au regard des objets centraux présentés

2 dans le tableau 1, ils sont fortement représentés sur l'axe 1 et l'axe 3. Ils mangeaient

3 majoritairement bio avant d'adhérer. Leurs habitudes n'ont pas changé. C'est d'ailleurs plutôt

4 parmi eux qu'on trouve ceux qui étaient déjà adhérents à des systèmes de paniers. Cette classe

5 typologique se caractérise de plus par des niveaux élevés d'engagement et de satisfaction

6 (satisfaction cumulée). Ils ont une bonne perception du point relais et de sa praticité. La

7 formule leur convient et leur proximité de processus avec le système ("en savoir plus sur les

8 conditions de production") est plus élevée que pour les autres groupes. Ce sont souvent des

9 couples avec enfants.

10 Après avoir validé la fiabilité de chaque variable du modèle, l'intention de se réabonner pour

11 les «expérimentés» s'écrit: Intention de se réabonner $=0,443$ engagement $+0,335$

12 satisfaction $+0,074$ proximité processus.

13 La figure 2 illustre ce modèle causal.

14 Figure 2 - Modèle causal des intentions de se réabonner des « expérimentés »

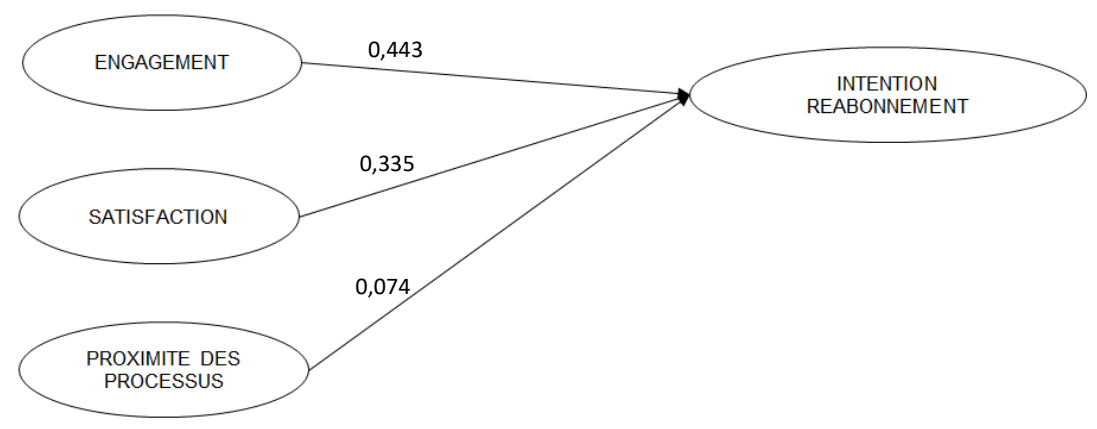

16 Le modèle causal montre que l'engagement et la satisfaction expliquent principalement 17 l'intention de rester.

18 En calculant la moyenne de chaque variable manifeste composant le modèle causal des 19 « expérimentés », nous obtenons le score d'intention de réabonnement suivant : 3,05/4,26 soit $2072 \%$ (écart de 1,21 - soit $28 \%$ ). Ce groupe est marqué par une intention de rester beaucoup 21 plus élevée que les deux autres et présente le plus faible écart $(1,21)$ à la note maximale 22 (4,26). Ce système se rapproche de la maximisation de la valeur perçue par ces abonnés. 
1 Groupe 3 - «les expérimentateurs » (265 individus). Un troisième groupe de 265 individus

2 émerge. Nommés «expérimentateurs », ils se caractérisent par un fort changement

3 d'habitudes alimentaires depuis leur adhésion aux PBS (fortement représenté sur l'axe 2 -

4 tableau 1). Ce groupe vit une expérience nouvelle. Les individus n'étaient pas consommateurs

5 de bio auparavant ; désormais ils déclarent particulièrement manger plus varié, plus équilibré,

6 faire davantage la cuisine, préparer de nouvelles recettes, découvrir de nouveaux produits,

7 consommer davantage de fruits et légumes et de produits bio en général. En revanche, ils ne

8 se sentent ni engagés ni proches de ce système (proximité axiologique). Ils viennent de

9 circuits de distribution conventionnels et les prix sont perçus comme élevés. Ce sont souvent

10 des célibataires ou couples sans enfant (valeur négative sur l'axe 6 - tableau 1).

11 Après avoir validé la fiabilité de chaque variable du modèle, l'intention de se réabonner pour

12 les «expérimentateurs »s'écrit: Intention de se réabonner $=0,406$ engagement $+0,349$

13 satisfaction $+0,126$ composition familiale $-0,122$ proximité axiologique $-0,094$ mangeait

14 déjà bio $+0,058$ confiance $+0,044$ proximité processus.

15 La figure 3 illustre ce modèle causal.

16 Figure 3 - Modèle des intentions de se réabonner des « expérimentateurs »

17

18

19

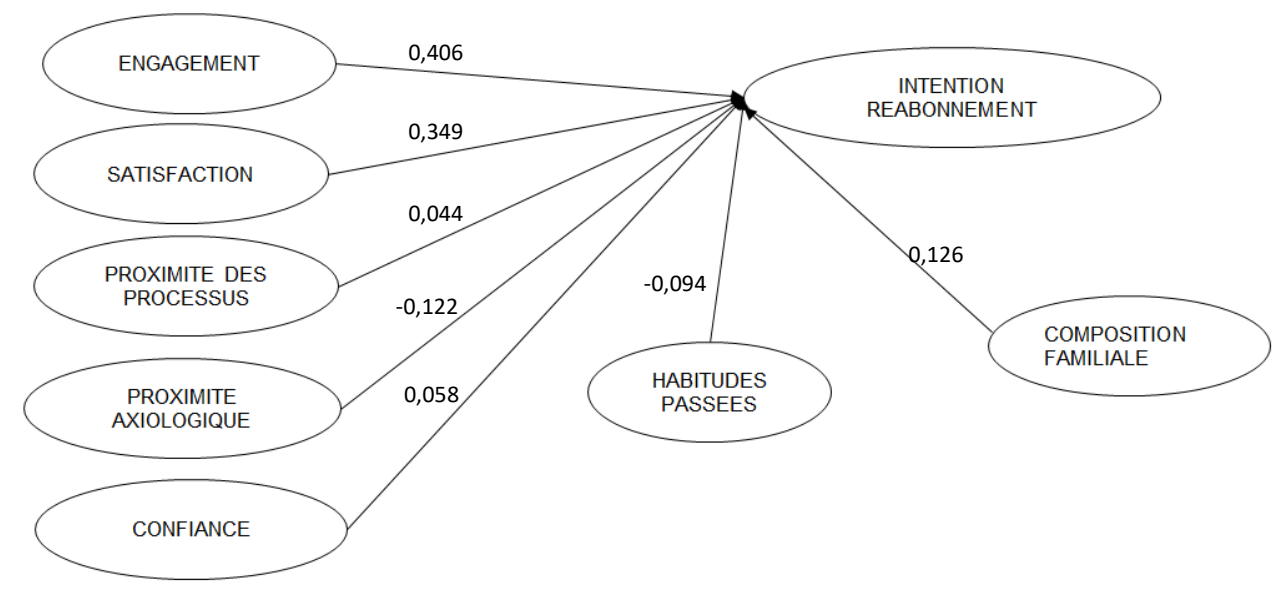

Logiciel : Xlstat PLSPM

L'engagement, la satisfaction contribuent là aussi prioritairement à former les intentions de réabonnement de ce groupe. Dans une moindre mesure, la composition familiale joue, ceux 
1 qui ont des enfants exprimant davantage une intention positive (rappelons que ce groupe a peu

2 d'enfants). La proximité axiologique et le fait d'être déjà mangeur de bio jouent négativement

3 (mais peu) sur les intentions de réabonnement.

4 En calculant la moyenne de chaque variable manifeste composant le modèle causal des

5 « expérimentateurs », nous obtenons le score d'intention de réabonnement suivant : 2,51/3,84 soit $65 \%$ (écart de 1,33 - soit $35 \%$ ). Ce groupe présente le plus faible score d'intention de se réabonner. L'écart à la valeur maximale $(3,84)$ est plus fort que celui du groupe des « expérimentés ».

9 Sur un plan sociétal, ce groupe est donc particulièrement intéressant car les PBS jouent un 10 rôle pédagogique. C'est un groupe en phase de transition; il est difficile de prévoir leur 11 réabonnement, pour eux aussi d'ailleurs - s'acculturer ou quitter (Dufeu et Ferrandi, 2013). Finalement, l'engagement et la satisfaction représentent les facteurs majeurs de l'intention comportementale (de réabonnement) des adhérents. Les changements (ou pas) d'habitudes de consommation permettent quant à eux de segmenter en trois groupes distincts les adhérents.

\section{Les changements d'habitudes discriminent les profils d'abonnés mais} n'expliquent pas leur prédisposition à s'engager durablement

À ce stade, nous cherchons à tester sur un plan causal le rôle des changements d'habitudes de consommation dans la construction d'un engagement durable (dépassant la simple approche comportementale de réabonnement).

La construction ad hoc de la variable composite d'engagement durable

Étudier la construction d'un lien durable entre un objet et un sujet rejoint le champ de la fidélité. Toutefois, le jeu de données de cette recherche nous amène à questionner prioritairement la notion d'engagement, variable déterminante de la fidélité et pour laquelle nous disposons d'items de mesure. C'est ainsi qu'à partir des données de cette recherche, nous pouvons tester une variable «composite » d'engagement durable (dépassant la simple 
1 variable d'intention de se réabonner). Nous composons empiriquement une variable

2 d'engagement durable autour des items de mesure affectifs (tirés des contributions de

3 Lacœuilhe, 2000) et conatifs (tirés de Cissé-Depardon et N'Goala, 2009) : «j'aurais du mal à

4 quitter les PBS »; «je suis attaché aux PBS»; «je reviendrais vers les PBS si je devais

5 cesser mon abonnement quelque temps ».

6 Les indicateurs $^{7}$ statistiques de validation des échelles de mesure confirment la fiabilité de 7 cette variable «composite» permettant ainsi le développement des tests confirmatoires 8 suivants.

10 Les changements (ou pas) d'habitudes alimentaires ne construisent pas

11 l'engagement durable

12 L'adhésion aux PBS provoque des changements importants chez les abonnés, selon leurs 13 dires : une grande majorité estime que leur adhésion a accru la quantité (65\% des répondants) 14 et la variété (78\%) de fruits et de légumes consommés. Ils déclarent quatre fois sur cinq avoir 15 découvert de nouveaux produits et apprécié ces découvertes, préparer de nouvelles recettes $16(88 \%)$ et passer plus de temps à cuisiner (61\%). Enfin, quatre sur cinq disent consommer 17 désormais davantage de produits écologiques ou biologiques en général et $93 \%$ déclarent 18 parler des PBS autour d'eux.

19 Ces consommateurs n'étaient généralement (à $95 \%$ ) pas adhérents de systèmes de paniers : 20 ils en expérimentent les contraintes sur un plan quotidien, liées à la saisonnalité de la 21 production locale. Découvrir de nouveaux produits ou, au moins, se voir livrer des produits 22 inhabituels nécessite pour eux de cuisiner beaucoup plus, d'apprendre de nouvelles recettes et 23 de manger différemment.

24 À notre connaissance, quelques recherches se sont intéressées aux changements d'habitudes 25 comportementales dans le cadre des produits bio et locaux (Dufeu et Ferrandi, 2013 ; Robert26 Demontrond, Beaudouin et Dabadie, 2017). Mais aucune ne s'est centrée sur la capacité explicative de ces facteurs à générer un lien durable envers le système de panier. Autrement

7 Alpha de Cronbach =0,762; Rho D.G =0,863. 
1 dit, aucune échelle de référence ne préexiste. C'est pourquoi, nous construisons une échelle

$2 a d$ hoc dont les items sont totalement tirés des développements empiriques de cette recherche.

3 L'échelle de changements d'habitudes alimentaires se construit par conséquent autour des

4 items suivants : «Je mange plus de fruits et légumes ; je mange plus équilibré ; je mange plus

5 varié/diversifié ; je cuisine plus souvent; je prépare de nouvelles recettes ».

6 Nous testons, par équations structurelles, le pouvoir explicatif de la dimension « changement 7 d'habitudes» vs «absence de changement d'habitudes» sur la variable composite

8 d'engagement durable. Les tableaux 2 et 3 présentent les résultats pour chaque test.

10 Tableau 2 : Test de l'échelle du changement d'habitudes sur la prédisposition à rester

\begin{tabular}{|c|c|}
\hline $\begin{array}{l}\text { L'échelle du changement d'habitudes se } \\
\text { compose empiriquement de : }\end{array}$ & $\begin{array}{l}\text { - Je mange plus de fruits et légumes. } \\
\text { - Je mange plus équilibré. } \\
\text { - Je mange plus varié/diversifié. } \\
\text { - Je cuisine plus souvent. } \\
\text { - Je prépare de nouvelles recettes. }\end{array}$ \\
\hline \multicolumn{2}{|c|}{$\begin{array}{l}\text { Les changements d'habitudes de consommation, de manière significative, ne construisent pas } \\
\text { la prédisposition à s'engager durablement }\left(\mathrm{R}^{2}=0,052\right) \text {. }\end{array}$} \\
\hline
\end{tabular}

12 Symétriquement, nous avons construit une échelle d'absence de changement d'habitudes 13 autour d'items de mesure issus du terrain de cette recherche : « je ne consomme pas plus de 14 produits biologiques et/ou écologiques; avant les paniers, aviez-vous l'habitude de 15 consommer des produits issus de l'agriculture biologique ? [Légumes et fruits]; avant les 16 paniers, aviez-vous l'habitude de consommer des produits issus de l'agriculture biologique ? 17 [Autres produits (pain, produits laitiers, viandes, céréales...)]». 
1 Tableau 3 : Test de l'échelle de l'absence de changement d'habitudes sur la prédisposition à 2 rester

\begin{tabular}{|c|c|}
\hline $\begin{array}{l}\text { L'échelle de l'absence de changement } \\
\text { d'habitudes se compose empiriquement de : }\end{array}$ & $\begin{array}{l}\text { - Je ne consomme pas plus de produits } \\
\text { biologiques et/ou écologiques. } \\
\text { - Avant les paniers, aviez-vous l'habitude de } \\
\text { consommer des produits issus de l'agriculture } \\
\text { biologique? [Légumes et fruits]. } \\
\text { - Avant les paniers, aviez-vous l'habitude de } \\
\text { consommer des produits issus de l'agriculture } \\
\text { biologique ? [Autres produits (pain, produits } \\
\text { laitiers, viandes, céréales...)]. }\end{array}$ \\
\hline \multicolumn{2}{|c|}{$\begin{array}{l}\text { L'absence de changement d'habitudes de consommation, de manière significative, ne } \\
\text { construit pas la prédisposition à s'engager durablement }\left(\mathrm{R}^{2}=0,034\right) \text {. }\end{array}$} \\
\hline
\end{tabular}

$4 \mathrm{Au}$ total, contrairement à ce que nous pouvions attendre, le changement d'habitudes 5 alimentaires ne prédispose pas à s'engager durablement. 


\section{Conclusion, implications et limites}

2 Les systèmes d'abonnement à des paniers alimentaires locaux se multiplient ces dernières

3 années mais sont confrontés de manière particulièrement sensible à une problématique

4 marketing classique, celle de la fidélité des adhérents. La présente recherche s'est penchée sur

5 ce sujet en s'appuyant sur le cas d'un système de paniers fruits et légumes bio et locaux en

6 Pays-de-la-Loire, les Paniers Bio Solidaires (PBS). Malgré les bons scores de satisfaction

7 obtenus en moyenne lors de sondages, les PBS constatent un renouvellement important de

8 leur portefeuille clients. Ce constat montre les limites d'une approche explicative centrée

9 exclusivement sur les cognitions et le traitement de la performance par moyennes (négligeant

10 ainsi les différences entre les abonnés). Ce travail s'est ainsi attaché, à partir de l'exploitation

11 de 825 réponses à un questionnaire envoyé aux adhérents, à révéler les spécificités des

12 abonnés à ce système de paniers permettant d'affiner l'appréciation de la performance de ce 13 système.

14 Cette recherche présente finalement quatre résultats majeurs constituant autant de contributions : (1) les abonnés à un système de paniers ne sont pas homogènes dans leurs habitudes alimentaires, leurs perceptions de l'offre et dans leurs intentions comportementales; (2) l'engagement et la satisfaction représentent les principaux facteurs déterminants de l'intention comportementale; (3) la satisfaction influence l'intention comportementale mais n'est pas pour autant facteur de rétention; (4) les changements

20 d'habitudes, quoique permettant de segmenter les abonnés des PBS, n'expliquent pas la 21 prédisposition à s'engager durablement.

22 (1) L'étude du profil des abonnés (825 au total) a tout d'abord révélé trois profils différents liés aux perceptions et surtout aux habitudes de consommation (les «expérimentés », les « expérimentateurs », les «conventionnels») dont Dufeu et Ferrandi (2013) montraient qu'elles jouaient un rôle central chez les Amapiens. À partir de modèles d'équations structurelles, nous avons en effet pu tester l'intention de réabonnement pour chaque profil et 27 calculer ainsi pour chacun un score d'intention de se réabonner. Nos résultats - affinés par 28 profil - permettent de proposer des actions ciblées et d'augmenter ainsi l'efficacité des programmes. 
1 Cette recherche montre en effet que le groupe «d'expérimentés » en matières de systèmes

2 alimentaires locaux affiche une intention de réabonnement plus élevée que la moyenne (et 3 inversement pour les moins expérimentés). Ils représentent par conséquent le cœur de cible 4 des PBS. Les systèmes de paniers devraient donc attacher une attention toute particulière aux actions donnant du sens, de la valeur symbolique à leur offre. Dans cette optique, il est certes nécessaire de maintenir une communication centrée sur la proposition alternative des PBS orientés bio, local et solidaire en vue de renforcer le sens donné à cette démarche de consommation des «expérimentés ». Mais les résultats suggèrent que cette communication « descendante » n'est pas suffisante (le niveau d'information concernant des attributs de

10 l'offre est satisfaisant pour la plupart des répondants). Il serait ici souhaitable d'inscrire la relation dans une démarche co-créatrice de valeur puisque ce cœur de cible partage les valeurs des PBS, se sent proche d'eux. Concrètement, l'abonné participerait et créerait avec les PBS en s'invitant par exemple dans le choix des produits proposés, en partageant son avis auprès d'une communauté reliée aux PBS (ce qui impliquerait de fonder ce groupe au préalable).

Concernant « les conventionnels» dont les intentions comportementales sont majoritairement reliées à leurs perceptions du système de distribution (qui se doit d'être rapide et pratique d'accès) et à la confiance en les PBS, nous recommandons aux PBS de :

- maintenir une vigilance sur les règles transactionnelles de la distribution : proposer « le bon produit, au bon endroit, dans les bonnes quantités» en vue de satisfaire les attentes fonctionnelles des adhérents ;

21 - communiquer sur les éléments qui rendent crédible et légitime l'offre proposée de manière à 22 renforcer la confiance dans le temps et détourner ainsi cette clientèle des circuits de 23 distribution classiques ;

24 - prendre un soin particulier dans la sélection des magasins partenaires qui servent de points 25 relais. En effet, si une majorité des répondants se dit satisfaite de ces derniers, la typologie 26 nous montre que cet aspect fonctionnel est discriminant. Les «conventionnels » ont des attentes particulièrement saillantes en la matière.

Le groupe des «expérimentateurs» se caractérise par un fort changement d'habitudes alimentaires depuis leur adhésion aux PBS. Ils n'étaient pas consommateurs de bio 
1 auparavant ; désormais ils déclarent particulièrement manger plus varié, plus équilibré, faire

2 davantage la cuisine, préparer de nouvelles recettes, découvrir de nouveaux produits etc. Ils

3 ne se sentent pas forcément proches des valeurs du système de paniers, c'est pourquoi il

4 conviendra de :

5 - accompagner cette transition en communiquant à cette cible les bénéfices de ce type de 6 consommation (communiquer sur les produits, les recettes, les bénéfices symboliques), 7 d'autant que ce groupe est dominé par des individus plutôt jeunes et sans enfant, susceptibles

8 d'être utilisateurs du site internet des PBS ;

9 - satisfaire les règles d'assortiment en proposant des produits frais, variés et bons pour la 10 santé, autrement dit, tenter d'élargir l'assortiment de paniers ;

11 - maintenir une ligne de prix raisonnables de manière à convertir durablement ces abonnés 12 issus des circuits de distribution classique, orientee vers les prix bas.

13 L'approfondissement de ce travail statistique, sur l'ensemble de l'échantillon, montre 14 cependant que les changements d'habitudes alimentaires ne prédisposent pas à un engagement 15 durable. Nous reviendrons sur ce point.

16 (2) Sur un plan théorique, ce travail, en explorant les leviers de la fidélité attitudinale dans le 17 cadre d'un système de paniers, s'inscrit dans le cumul des connaissances dédiées à ce système 18 commercial peu étudié (les contributions passées peu nombreuses se sont centrées davantage 19 sur les perceptions des adhérents). Il montre que parmi les leviers que la revue de littérature 20 avait permis d'identifier (engagement, satisfaction, confiance, proximités et comportements 21 de consommation, pour l'essentiel), l'engagement est la variable qui contribue prioritairement 22 et directement à prévoir les intentions comportementales. L'engagement, tel qu'il est mesuré 23 dans cette recherche, a une double dimension affective (souvent assimilée à l'attachement) et 24 conative. Les PBS semblent par conséquent très marqués par ces dimensions. Ce résultat 25 habituel lorsqu'il s'agit des systèmes alimentaires locaux mais aussi plus largement du 26 marketing relationnel (Lay-Hwa Bowden, 2009 ; Raïes et Gavard-Perret, 2011), montre que les consommateurs de paniers ne sont pas fondamentalement différents des autres. Ce résultat constitue la seconde contribution de ce travail. 
1 (3) Le troisième résultat qui pourra être retenu pour les recherches futures est que, si une forte

2 satisfaction d'ensemble ne garantit certes pas le maintien de la population d'adhérents

3 (rétention), le niveau de satisfaction envers les variables du mix marketing (produits, paniers

4 et prix) détermine l'intention comportementale de réabonnement. Ceci est vrai quel que soit le profil des consommateurs. Jamais réellement démenti dans les recherches précédentes citées dans cet article, ce résultat confirme que l'achat éthique ne conduit pas le consommateur à ignorer son intérêt sur un plan fonctionnel. Ces éléments rejoignent les contributions passées reliées au marketing de la marque, où les bénéfices de consommation orientés «valeur » construisent de manière durable une relation avec le client (Keller, 1993 ; Bennett et RundleThiele, 2004). En cela, le système de paniers ne diffère pas théoriquement du champ classique des produits de grande consommation. Il semblerait donc que les résultats de notre recherche renvoient les PBS davantage dans le champ du marketing relationnel en général que dans celui de la consommation responsable fortement engagée (Amap, magasins associatifs etc.) Ce résultat permet de préciser et d'affiner le cadre de réflexion de ce système commercial ; en cela il constitue la troisième contribution de cette recherche.

Parallèlement, une composante relationnelle qui importe habituellement beaucoup dans les travaux sur les systèmes alimentaires alternatifs joue un rôle faible dans notre étude : il s'agit des différentes dimensions de la confiance (Gurviez et Korchia, 2002). Les PBS constituent une forme de marketing relationnel assez poussée et le lien entre la confiance et l'attitude (un

20 lien faible pour deux des trois groupes) pouvait être attendu à un niveau plus élevé (Merle et 21 Piotrowski, 2012 ; Aissaoui, Bueno Merino et Grandval, 2017 ; Dufeu et Ferrandi, 2013). Or, la confiance joue un rôle presque nul en ne contribuant qu'à hauteur de $0,05 \%$ dans le modèle général. Cela vient probablement du fait qu'une confiance déclarée très élevée est quasiment unanime parmi les répondants (moyenne à 4,7/5). Donc cette variable n'est pas discriminante.

Engagement et satisfaction détermineraient donc les attitudes? Rien de nouveau sous le soleil... des paniers ? Il semblerait. Pour se faire un avis, il est utile d'aborder le dernier résultat (4), celui du rôle (incertain ici) des changements d'habitudes de consommation. Rappelons que les abonnés déclarent dans leur grande majorité avoir fortement modifié leurs comportements alimentaires depuis leur adhésion (ils cuisinent davantage et différemment, 
1 mangent plus de fruits et légumes, etc.). Curtis, Allen et Ward (2015) trouvent un résultat 2 similaire dans le cas des CSA (Community Supported Agriculture, l'équivalent des Amap) 3 aux États-Unis. Rappelons également que ces changements discriminent la population des 4 répondants dans la typologie issue de la classification hiérarchique. Le lien statistique entre 5 l'attitude des abonnés et leur expérience en matière de consommation de fruits et légumes 6 locaux et saisonniers pouvait donc, en première analyse, nous inciter à penser que c'est là que se jouait la fidélisation. Mais un test de causalité n'a pas permis de confirmer cette hypothèse. Il est difficile de conclure de manière plus générale à ce stade mais gageons qu'avec un résultat inverse nous nous serions empressés d'émettre l'hypothèse qu'une acculturation serait nécessaire au maintien dans le système (Curtis, Allen et Ward, 2015 ; Dufeu et Ferrandi, 2013)! Nous pouvons émettre l'hypothèse selon laquelle ceux qui vivent ces changements d'habitude négativement (essentiellement comme une contrainte) compensent statistiquement ceux qui les vivent comme une expérience agréable, mais nos données actuelles ne nous permettent pas de tester cette hypothèse. D'autres travaux seront utiles pour l'examen de ce lien entre évolutions comportementales et fidélisation dans le cadre des systèmes de paniers. Nous avons ainsi engagé un partenariat avec les PBS pour conduire de nouvelles enquêtes qui permettront d'aborder cette question.

Malgré les incertitudes qui subsistent, et les limites inhérentes à toute recherche empirique, ces résultats nous suggèrent quelques implications managériales.

La première concerne la manière dont les enquêtes de satisfaction sont mobilisées par les acteurs. Les gestionnaires des PBS avaient auparavant conçu une enquête annuelle très complète concernant la valeur perçue; mais la prise en compte des attentes des consommateurs n'a pas produit de résultats spectaculaires en matière de fidélisation. Cette recherche teste, mesure et confirme les travaux passés en lien avec l'insuffisance du pouvoir explicatif des dimensions cognitives dans un modèle d'intention comportementale. Elle montre par ailleurs l'importance de segmenter les profils de consommateurs sur des critères autres que les critères historiques de segmentation tels que les indicateurs sociodémographiques. Les segmentations obtenues ici permettent de révéler précisément toutes les subtilités de cette problématique : il n'y a pas un profil d'abonnés mais trois. On retrouve ici la pluralité culturelle qui caractérise le mouvement amapien selon Robert- 
1 Demontrond, Beaudouin et Dabadie (2017). S'appuyer sur la moyenne des variables du

2 sondage est donc totalement inutile pour apprécier justement la performance du système. En 3 cela, ce travail permet d'aider la prise de décision des PBS, de mieux comprendre les 4 perceptions, les attentes et les comportements de leurs abonnés et d'affiner leur ciblage en trois groupes distincts. Plus précisément, cette recherche révèle que le groupe des «expérimentés » présente un score d'intention de se réabonner supérieur aux deux autres groupes et un écart à la valeur maximale le plus faible. En cela, ils peuvent être considérés comme le cœur de cible des PBS. À l'inverse, les autres groupes «conventionnels» et «expérimentateurs» représentent des consommateurs moins habitués à ce type de consommation bio local contraignant et sont moins motivés à rester.

La seconde implication est liée au fait que ces résultats nous montrent que, même alternatif et militant, un système de paniers doit tenir compte des enseignements du marketing relationnel en général. Nous l'avons dit, les variables déterminant la fidélité des adhérents ne sont pas particulièrement spécifiques. Plus encore, pour un groupe en particulier d'adhérents, les attentes fonctionnelles semblent jouer un rôle primordial - une forme de prérequis de consommation semblable à toute offre commerciale classique.

17 À ce stade, l'ensemble de nos résultats confrontés aux recherches précédentes (notamment sur 18 les Amaps) pourrait nous conduire à formuler avec d'infinies précautions l'hypothèse suivante: les consommateurs d'un système de panier tel que les PBS ressembleraient finalement davantage à des consommateurs de circuits conventionnels qu'à des amapiens.

21 Dans ce cas, la question du rôle exercé par l'adhérent (passif dans le cas des PBS, actif dans le cas des Amap) pourrait s'avérer déterminante.

Cette recherche revêt un certain nombre de limites, au premier rang desquelles figure classiquement un biais de sélection, puisque les réponses sont volontaires; mais le taux de réponse est très élevé (près de $40 \%$ ) ce qui limite ce biais. Une autre limite de ce travail repose sur la mesure des intentions comportementales et non des comportements réels. Cela renvoie cependant au concept de fidélité attitudinale qui est un objet d'étude en soi, distinct de la fidélité comportementale même si les deux concepts sont liés. Les auteurs travaillent désormais sur la production de données à même de mesurer la fidélité comportementale aux 
dma190087 R3

1 PBS. Les comportements réels ainsi identifiés permettront d'élargir le spectre de cette 2 recherche en confrontant l'intention comportementale au comportement effectif (rester ou 3 partir) et d'expliquer finalement la rétention à un système de paniers (approche précisée par 4 Crié, 1996). 


\section{Bibliographie}

2 Aissaoui S., Bueno Merino P. et Grandval S. (2017), Les antécédents de la confiance dans la coopération amapienne, Revue internationale P.M.E., 30(1): 121-154.

Babin J., Darden W. R. et Mitch G. (1994), Work and/or fun: measuring hedonic and utilitarian shopping value, Journal of Consumer Research, 20(4): 644-656.

Belaid S., Ben Mrad S., Lacœuilhe J. et Turki Tlili H. (2017), Des consommateurs hédonistes, symboliques ou fonctionnels? Développement et validation d'une échelle des bénéfices rattachés aux marques dans le cadre des marchés émergents : le cas de la Tunisie, Revue Française du Marketing, 261(3): 65-79.

Bennett R. et Rundle-Thiele S. (2004), Examining the satisfaction-loyalty relationship, Journal of Services Marketing, 18(7): 514-523.

12 Bergadaà M. et Del Bucchia C. (2009), La recherche de proximité par le client dans le secteur 13 de la grande consommation alimentaire, Revue Management et Avenir, 21(1): 121-135.

Bove, L. et Mitzifiris, B. (2007), Personality traits and the process of store loyalty in a transactional prone context, Journal of Services Marketing, 21(7): 507-19.

Chaudhuri A. et Holbrook M. B. (2001), The chain of effects from brand trust and brand affect to brand performance: the role of brand loyalty, Journal of Marketing, 65(2): 82-93.

Cissé-Depardon K. et N'Goala G. (2009), Les effets de la satisfaction, de la confiance et de l'engagement vis-à-vis d'une marque sur la participation des consommateurs à un boycott, Recherche et Applications en Marketing, 24(1): 43-67.

Crié D. (1996), Rétention de clientèle et fidélité des clients, Décisions Marketing, (7): 25-30.

Curtis K. R., Allen K. et Ward R. A. (2015), Food consumption, attitude, and behavioral change among CSA members: a northern Utah case study, Journal of Food Distribution Research, 46(2): 3-16.

Dufeu I. et Ferrandi, J.-M. (2013), Les ressorts de l'engagement dans une forme particulière d'échange collaboratif entre producteur et consommateurs: les AMAP, Décisions Marketing, (72): 157-178.

Frisou J. (2004), Le contrôle du comportement de fidélité: premiers jalons pour une théorie dynamique et éclectique, Recherche et Applications en Marketing, 19(4): 117-118. 
1 Gurviez P. et Korchia M. (2002), Proposition d'une échelle de mesure multidimensionnelle de

2 la confiance dans la marque, Recherche et Applications en Marketing, 17(3): 41-62.

3 Hérault-Fournier C., Merle A. et Prigent-Simonin A.-H. (2014), Diagnostiquer la proximité

4 perçue en vente directe de produits alimentaires, Décisions Marketing, (73): 85-104.

Jacoby J. et Kyner D.B. (1973), Brand loyalty vs. repeat purchasing behavior, Journal of Marketing Research, 30(1): 118-137.

Jones T.O. et Sasser W.E. (1995), Why satisfied customers defect?, Harvard Business Review, 73(6): 88-99.

Keller K. L. (1993), Conceptualising, measuring and managing customer-based brand equity, Journal of Marketing, 57(1), 1-22.

Labbé-Pinlon B., Lombart C. et Louis D. (2016), Impact de la proximité perçue d'un magasin sur la fidélité des clients : le cas des magasins d'enseignes alimentaires de proximité, Management \& Avenir, 84(2): 73-94.

14 Lacœuilhe J. (2000), L'attachement à la marque: proposition d'une échelle de mesure, Recherche et Applications en Marketing, 15(4): 61-77.

Lay-Hwa Bowden J. (2009), The process of customer engagement: a conceptual framework, Journal of Marketing Theory and Practice, 17(1): 63-74.

Lichtlé M.-C. et Plichon V. (2008), Mieux comprendre la fidélité des consommateurs, Recherche et Applications en Marketing, 23(4): 121-141.

Merle A. et Piotrowski M. (2012), Consommer des produits alimentaires locaux : comment et 21 pourquoi ?, Décisions Marketing, (67): 37-48.

Merle A., Hérault-Fournier C. et Werle C. (2016), Les effets de la mention d'origine géographique locale sur les perceptions alimentaires, Recherche et Applications en Marketing, 31(1); 28-45.

Michael D. C., Christopher G., Tzu-Hui K. et Michelle C. (2008), An empirical analysis of customer satisfaction in international air travel, Innovative Marketing, 4(2): 49-62.

Morgan R. M. et Hunt S. D. (1994), The commitment-trust theory of relationship marketing, Journal of Marketing, 58(3): 20-38.

29 Mundler P. (2013), Le prix des paniers est-il un frein à l'ouverture sociale des AMAP ? Une 30 analyse des prix dans sept AMAP de la Région Rhône-Alpes, Économie Rurale, (336): 3-19. 
1 Oliver, R. L. (1999), Whence customer loyalty?, Journal of Marketing, 63(4): 33-44

2 Orth U. R. et De Marchi, R. (2007), Understanding the relationships between functional, 3 symbolic, and experiential brand beliefs, product experiential attributes, and product 4 schema: advertising-trial interactions revisited, Journal of Marketing Theory and Practice, 5 15(3): 219-233.

6 Park C. W., Jaworski B. J. et McInnis D. J. (1986), Strategic brand concept-image 7 management », Journal of Marketing, 50(3): 324-340.

8 Raïes K. et Gavard-Perret M.-L. (2011), Intention de fidélité à la marque des participants à 9 une communauté virtuelle de marque: le rôle dual de l'engagement, Recherche et 10 Applications en Marketing, 26(3); 23-43.

11 Rivière A. et Mencarelli R. (2012), Vers une clarification théorique de la notion de valeur perçue en marketing, Recherche et Applications en Marketing, 27(3): 97-123.

Robert-Demontrond P., Beaudouin V. et Dabadie I. (2017), Diversité, conflictualité, 14 complémentarité des visions du monde: une investigation anthropologique de la consommation en Amap, Recherche et Applications en Marketing, 32(4): 37-57.

16 Strizhakova Y., Coulter R. A. et Price L. L. (2008), The meanings of branded products: a cross-national scale development and meaning assessment, International Journal of Research in Marketing, 25(2): 82-93.

Sweeney J. C. et Soutar G. N. (2001), Consumer perceived value: The development of a multiple item scale, Journal of Retailing, 77(2): 203-220.

21 Tucker W. T. (1964), The development of brand loyalty, Journal of Marketing Research, 22 1(3): 32-35. 


\section{Annexe 1a-Procédure des tests de fiabilité des échelles de mesure}

Les construits de chaque modèle sont réflectifs ; en cela, nous suivons la procédure proposée par Churchill (1979) portant sur l'examen de la fiabilité et de la validité convergente des construits latents. Les analyses confirmatoires ont été menées à l'aide du logiciel Xlstat PLSPM (basé sur la technologie PLEASURE ${ }^{8}$ ) fondé sur la modélisation Path-PLS (Tenenhaus et al., 2005).

La fiabilité des mesures de construits réflectifs est usuellement appréciée par l'alpha de Cronbach qui mesure la cohérence interne entre les différents items de mesure. Un autre indicateur, le Rhô de Jöreskog, est souvent utilisé en complément de l'alpha de Cronbach. L'intérêt de ce dernier est d'intégrer le terme d'erreur dans le calcul de la cohérence interne (Chin, 1998). Les résultats obtenus montrent que toutes les échelles sont fiables et mesurent bien ce qu'elles ont pour but de mesurer (annexe 1b).

Ensuite, valider ${ }^{9}$ le modèle structurel revient à tester la qualité des relations causales entre les variables latentes du modèle et à identifier le poids ${ }^{10}$ de chacune des variables explicatives dans la formation de ce dernier. Pour ce faire, plusieurs indicateurs statistiques (Gof, AVE et $\mathrm{R}^{2}$ ) sont mobilisés et détaillés en annexe $1 \mathrm{c}$.

Chin W. W. (1998), Issue and opinion on structural equation, MIS Quarterly, 22(1):7-16.

Churchill G. A. (1979), A paradigm for developing better measures of marketing constructs, Journal of Marketing Research, 16(1): 64-73

Tenenhaus M., Vinzi V.E., Chatelin Y. et Lauro C. (2005), PLS path modeling, Computational Statistics and Data Analysis, 48(1): 59-205.

\footnotetext{
8 Partial LEAst Squares strUctural Relationship Estimation. Ce logiciel a été développé par Chatelin Y. M., Esposito Vinzi V., Lauro C. et Tenenhaus M.

9 Les analyses menées en vue de valider le modèle structurel sont là encore réalisées à l'aide du logiciel Xlstat PLSPM.

10 Le poids de chaque variable explicative est illustré par la valeur du chemin causal (path-coefficient).
} 
Annexe 1b - Structure et fiabilité des échelles évaluées par échelle de Likert en 5 échelons

\begin{tabular}{|c|c|c|c|}
\hline Variable latente & Variables manifestes & $\begin{array}{l}\text { Alpha de } \\
\text { Cronbach }\end{array}$ & $\begin{array}{c}\text { Rhô de } \\
\text { D.G. } \\
\text { (ACP) }\end{array}$ \\
\hline \multirow{5}{*}{ POINT RELAIS } & Accueil & \multirow{5}{*}{0,824} & \multirow{5}{*}{0,877} \\
\hline & Horaires & & \\
\hline & Temps d'attente & & \\
\hline & Qualité des produits ou services proposés & & \\
\hline & L'accessibilité du lieu (parking, facile à trouver, ...) & & \\
\hline PRIX & Niveau de prix perçu & & \\
\hline \multirow[t]{3}{*}{ CONFIANCE } & J'ai confiance en la qualité des produits & \multirow{3}{*}{0,659} & \multirow{3}{*}{0,815} \\
\hline & Les PBS sont à mon écoute & & \\
\hline & Les produits sont sans danger & & \\
\hline \multirow{2}{*}{$\begin{array}{l}\text { PROXIMITÉ } \\
\text { RELATIONNELLE }\end{array}$} & Mieux connaître les producteurs & \multirow{2}{*}{0,755} & \multirow{2}{*}{0,891} \\
\hline & Rencontrer les producteurs & & \\
\hline
\end{tabular}


dma190087 R3

\begin{tabular}{|c|c|c|c|}
\hline Variable latente & Variables manifestes & $\begin{array}{l}\text { Alpha de } \\
\text { Cronbach }\end{array}$ & $\begin{array}{c}\text { Rhô de } \\
\text { D.G. } \\
\text { (ACP) }\end{array}$ \\
\hline \multirow{9}{*}{ PRODUITS } & Gestion de l'abonnement & \multirow{9}{*}{0,766} & \multirow{9}{*}{0,828} \\
\hline & Quantité & & \\
\hline & Diversité & & \\
\hline & Fréquence & & \\
\hline & Présentation & & \\
\hline & Goût & & \\
\hline & Proportion dans les paniers & & \\
\hline & Fraicheur & & \\
\hline & Tenue des produits & & \\
\hline
\end{tabular}




\begin{tabular}{|c|c|c|c|}
\hline Variable latente & Variables manifestes & $\begin{array}{l}\text { Alpha de } \\
\text { Cronbach }\end{array}$ & $\begin{array}{c}\text { Rhô de } \\
\text { D.G. (ACP) }\end{array}$ \\
\hline \multirow{3}{*}{$\begin{array}{l}\text { PROXIMITÉ } \\
\text { PROCESSUS }\end{array}$} & Je connais le mode de production des produits PBS & \multirow{3}{*}{0,697} & \multirow{3}{*}{0,833} \\
\hline & $\begin{array}{l}\text { J'ai accès aux informations concernant la production, la préparation et } \\
\text { la distribution des paniers }\end{array}$ & & \\
\hline & Je sais d'où proviennent les fruits et légumes des PBS & & \\
\hline \multirow{3}{*}{$\begin{array}{l}\text { PROXIMITÉ } \\
\text { IDENTITAIRE }\end{array}$} & Je partage plus de valeurs avec les PBS que... & \multirow{3}{*}{0,705} & \multirow{3}{*}{0,836} \\
\hline & Je connais les valeurs des PBS & & \\
\hline & Mes valeurs sont les mêmes que celles des PBS & & \\
\hline \multirow{4}{*}{$\begin{array}{l}\text { ENGAGEMENT/ } \\
\text { ATTACHEMENT/ } \\
\text { FIDÉLITÉ } \\
\text { ATTITUDINALE }\end{array}$} & Je suis attaché(e) aux PBS & \multirow{4}{*}{0,707} & \multirow{4}{*}{0,822} \\
\hline & Je parle des PBS autour de moi... & & \\
\hline & Je trouverais difficile de quitter les PBS & & \\
\hline & $\begin{array}{l}\text { Je reviendrais vers les PBS si je devais cesser mon abonnement } \\
\text { quelque temps (voyage, mutation...) }\end{array}$ & & \\
\hline INTENTION & $\begin{array}{l}\mathrm{Si} \text { votre abonnement est toujours en cours, pensez-vous vous } \\
\text { réabonner l'année prochaine? }\end{array}$ & & \\
\hline
\end{tabular}

La fiabilité de chaque échelle de mesure est confirmée puisque l'alpha de Cronbach et le Rhô de Dillon-Goldstein sont systématiquement supérieurs à $0,7$. 


\section{Annexe 1c - Indicateurs statistiques de validation du modèle causal}

\begin{tabular}{|l|l|}
\hline \multicolumn{1}{|c|}{ Indicateurs statistiques } & \multicolumn{1}{|c|}{ Seuil d'acceptation recommandé } \\
\hline $\begin{array}{l}\text { Le Goodness-of-Fit (GoF) mesure le pourcentage de } \\
\text { variance/covariance expliquée par le modèle (ce qui rejoint les } \\
\text { considérations du coefficient de détermination). Il convient de } \\
\text { s'intéresser plus particulièrement aux GoF du modèle interne } \\
\text { (modèle de structure) et du modèle externe (modèle de } \\
\text { mesure) ainsi qu'au GoF relatif. }\end{array}$ & $\begin{array}{l}\text { L'ensemble des indicateurs de } \\
\text { Goodness-of-Fit doit être supérieur à 0,9 } \\
\text { (Roussel et al., 2002). }\end{array}$ \\
\hline $\begin{array}{l}\text { L'AVE (Average Variance Extracted) ou variance moyenne } \\
\text { extraite, mesure la variance capturée par les construits. }\end{array}$ & $\begin{array}{l}\text { L'AVE doit être supérieure à 0,5 } \\
\text { (Fornell et Larcker, 1981) }\end{array}$ \\
\hline $\begin{array}{l}\text { Le coefficient de détermination (R²) indique la qualité de } \\
\text { l'ajustement global du modèle. Il indique le pouvoir explicatif } \\
\text { du modèle, autrement dit, le pourcentage de variance restituée } \\
\text { par les variables explicatives/indépendantes. }\end{array}$ & $\begin{array}{l}\text { Le coefficient de détermination doit être } \\
\text { supérieur à 0,5 (Tenenhaus, 2007). }\end{array}$ \\
\hline
\end{tabular}

Fornell C. et Larcker D.F. (1981), Structural equation models with unobservable variables and measurement error, Journal of Marketing Research, 18(3): 39-50.

Roussel P., Durrieu F., Campoy E. et El Akremi A. (2002), Méthodes d'équations structurelles : Recherche et applications en gestion, Paris, Economica.

Tenenhaus M. (2007), Statistique - Méthodes pour décrire, expliquer et prévoir, Paris, Dunod. 
Annexe 2 - Dendrogramme des trois profils d'abonnés

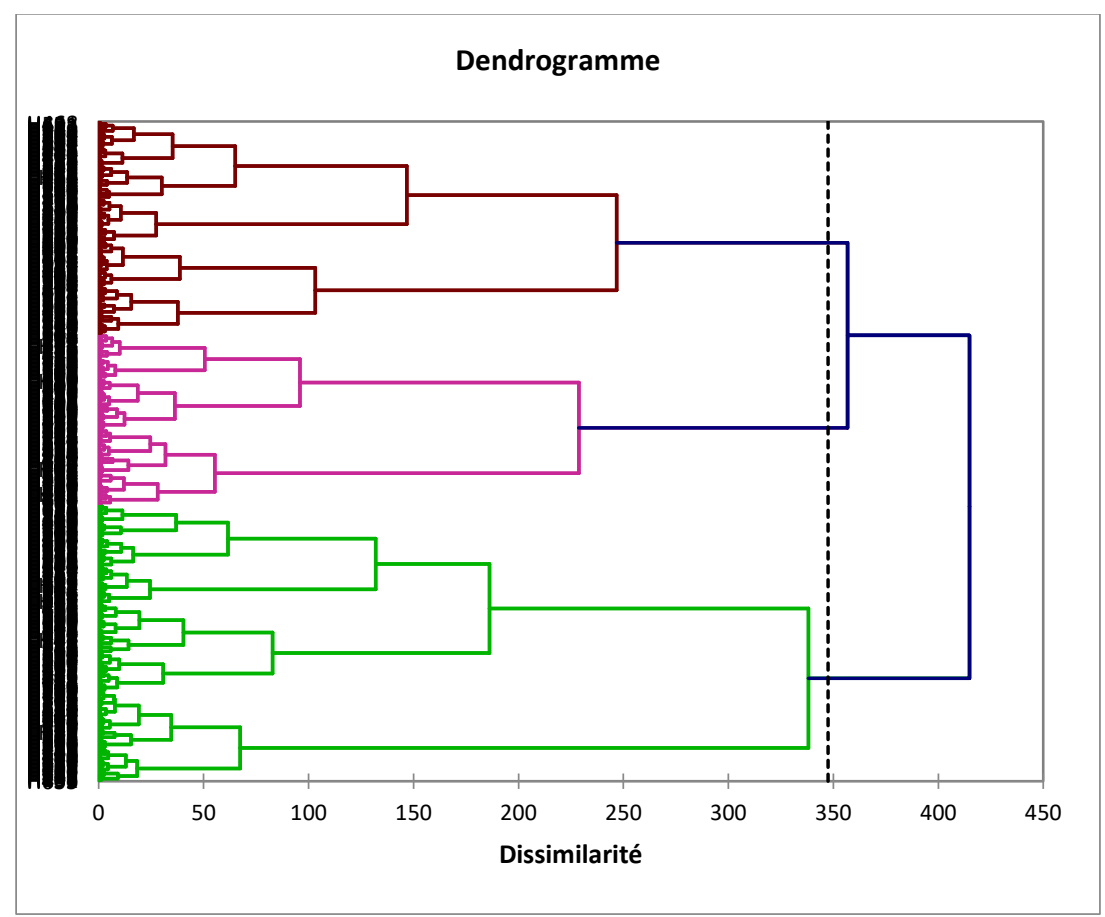

Logiciel : Xlstat 